\title{
Interactive comment on "Greenland climate simulations show high Eemian surface melt" by Andreas Plach et al.
}

\section{Andreas Plach et al.}

andreas.plach@gmail.com

Received and published: 13 November 2020

We would very much like to thank Anonymous Referee \#1 for the constructive comments. Please find our responses attached as a supplement.

Please also note the supplement to this comment:

https://cp.copernicus.org/preprints/cp-2020-101/cp-2020-101-AC1-supplement.pdf

Interactive comment on Clim. Past Discuss., https://doi.org/10.5194/cp-2020-101, 2020. 